\title{
An approximation approach for free space wave propagation
}

\begin{abstract}
Modern High technology live style demand an exhaustive used of electromagnetic devices, whether in civilian or military purposes. Among the applications of current interest include those related to communications. Advancement in Computer technology has shifted design of pilot product from ñclassical trial and erroròmethod to simulation method. This scenario has ñzoom-inò the essential of numerical simulation in most science and technology product research and development. Numerical method accelerates and facilitates research and development in the fields. Wireless communication technology has increase the need of ñsoftò tools to facilitate research and development in the area. A powerful algorithm that popularly used to simulate electromagnetic problem via Maxwell equations in time-domain is the well-known Finite-Difference Time-Domain (FDTD). Albeit FDTD is extraordinary method, it needs a long processing time to simulate problem. In our previous research, we have developed a method called High Speed Low Order Finite Difference Time Domain (HSLO-FDTD). The method is faster than the conventional FDTD but the accuracy is poorer. In this paper, we propose a new algorithm with high order characteristic. The efficiency of the new algorithm is tested and compared with the efficiency of high order FDTD method by some numerical experiment. Experimental result shows that the method is faster and has higher accuracy than the higher order FDTD method.
\end{abstract}

Keyword: Wave propagation; Maxwell equations; Finite difference time domain; Weighted arithmetic mean scheme 